Commun. Korean Math. Soc. 28 (2013), No. 3, pp. 635-642

http://dx.doi.org/10.4134/CKMS.2013.28.3.635

\title{
PARAMETRIC OPERATIONS FOR TWO FUZZY NUMBERS
}

\author{
Jisoo Byun AND Yong SiK Yun
}

\begin{abstract}
There are many results on the extended operations of two fuzzy numbers based on the Zadeh's extension principle. For the calculation, we have to use existing operations between two $\alpha$-cuts. In this paper, we define parametric operations between two $\alpha$-cuts which are different from the existing operations. But we have the same results as the extended operations of Zadeh's principle.
\end{abstract}

\section{Introduction}

In a fuzzy set theory, various types of operations between two fuzzy sets have been defined and studied. One of the most famous operation is the extended operations and there are many results on the extended operations of two fuzzy numbers based on the Zadeh's extension principle. Especially, many results of the extended algebraic operations between two triangular fuzzy numbers are very well-known.

In this paper, we prove that there exists some function (called the parametric $\alpha$-function) which characterizes the $\alpha$-cuts of fuzzy number. Using the parametric $\alpha$-functions, we define parametric operations between two $\alpha$-cuts. And then we define parametric operations between two fuzzy numbers. Finally we get the same results for extended addition, extended subtraction, extended multiplication and extended division between two fuzzy numbers.

\section{Preliminaries}

Let $X$ be a set, $A$ be a fuzzy set in $X$ and $\mu_{A}(x)$ be the membership function of $A$.

Definition 2.1. For $\alpha \in[0,1]$, the set $A_{\alpha}=\left\{x \in X \mid \mu_{A}(x) \geq \alpha\right\}$ is said to be the $\alpha$-cut of a fuzzy set $A$.

Received July 20, 2012.

2010 Mathematics Subject Classification. 47N30.

Key words and phrases. extended operation, parametric operation, continuous fuzzy number.

The first author was supported by Kyungnam University research fund, 2012.

(C)2013 The Korean Mathematical Society 
Then the followings are well known. The membership function of a fuzzy set $A$ can be expressed in terms of the characteristic functions of its $\alpha$-cuts according to the formula

$$
\mu_{A}(x)=\sup _{\alpha \in(0,1]} \min \left(\alpha, \mu_{A_{\alpha}}(x)\right),
$$

where

$$
\mu_{A_{\alpha}}(x)= \begin{cases}1, & x \in A_{\alpha} \\ 0, & \text { otherwise }\end{cases}
$$

Definition 2.2. A fuzzy set $A$ is convex if

$$
\mu_{A}\left(\lambda x_{1}+(1-\lambda) x_{2}\right) \geq \min \left(\mu_{A}\left(x_{1}\right), \mu_{A}\left(x_{2}\right)\right)
$$

for all $x_{1}, x_{2} \in X$ and $\lambda \in[0,1]$.

If we apply the extension principle to algebraic operations for fuzzy sets, we have the following definitions for extended operations.

Definition 2.3 ([3]). The extended addition, extended subtraction, extended multiplication and extended division are defined by

(1) extended addition $A(+) B$ :

$$
\mu_{A(+) B}(z)=\sup _{z=x+y} \min \left\{\mu_{A}(x), \mu_{B}(y)\right\}, x \in A, y \in B .
$$

(2) extended subtraction $A(-) B$ :

$$
\mu_{A(-) B}(z)=\sup _{z=x-y} \min \left\{\mu_{A}(x), \mu_{B}(y)\right\}, x \in A, y \in B .
$$

(3) extended multiplication $A(\cdot) B$ :

$$
\mu_{A(\cdot) B}(z)=\sup _{z=x \cdot y} \min \left\{\mu_{A}(x), \mu_{B}(y)\right\}, x \in A, y \in B .
$$

(4) extended division $A(/) B$ :

$$
\mu_{A(/) B}(z)=\sup _{z=x / y} \min \left\{\mu_{A}(x), \mu_{B}(y)\right\}, x \in A, y \in B .
$$

Remark 2.4 ([1]). Let $A$ and $B$ be fuzzy sets and $A_{\alpha}=\left[a_{1}^{(\alpha)}, a_{2}^{(\alpha)}\right]$ and $B_{\alpha}=$ $\left[b_{1}^{(\alpha)}, b_{2}^{(\alpha)}\right]$ be the $\alpha$-cuts of $A$ and $B$, respectively. Then the $\alpha$-cuts of $A(+) B$, $A(-) B, A(\cdot) B$ and $A(/) B$ can be calculated as follows.

(1) $(A(+) B)_{\alpha}=A_{\alpha}(+) B_{\alpha}=\left[a_{1}^{(\alpha)}+b_{1}^{(\alpha)}, a_{2}^{(\alpha)}+b_{2}^{(\alpha)}\right]$.

(2) $(A(-) B)_{\alpha}=A_{\alpha}(-) B_{\alpha}=\left[a_{1}^{(\alpha)}-b_{2}^{(\alpha)}, a_{2}^{(\alpha)}-b_{1}^{(\alpha)}\right]$.

(3) $(A(\cdot) B)_{\alpha}=A_{\alpha}(\cdot) B_{\alpha}=\left[a_{1}^{(\alpha)} b_{1}^{(\alpha)}, a_{2}^{(\alpha)} b_{2}^{(\alpha)}\right]$.

(4) $(A(/) B)_{\alpha}=A_{\alpha}(/) B_{\alpha}=\left[a_{1}^{(\alpha)} / b_{2}^{(\alpha)}, a_{2}^{(\alpha)} / b_{1}^{(\alpha)}\right]$.

In case $X=\mathbb{R}$, there are many more definite results.

Definition 2.5 ([4]). A fuzzy number $A$ is a convex fuzzy set on $\mathbb{R}$ such that

(1) there exists unique $x \in \mathbb{R}$ with $\mu_{A}(x)=1$,

(2) $\mu_{A}(x)$ is piecewise continuous. 
Definition 2.6. A triangular fuzzy number on $\mathbb{R}$ is a fuzzy number $A$ which has a membership function

$$
\mu_{A}(x)= \begin{cases}0, & x<a_{1}, \quad a_{3} \leq x, \\ \frac{x-a_{1}}{a_{2}-a_{1}}, & a_{1} \leq x<a_{2}, \\ \frac{a_{3}-x}{a_{3}-a_{2}}, & a_{2} \leq x<a_{3},\end{cases}
$$

where $a_{i} \in \mathbb{R}, i=1,2,3$.

The above triangular fuzzy number is denoted by $A=\left(a_{1}, a_{2}, a_{3}\right)$. In the calculations of algebraic operations for two fuzzy numbers, the concept of $\alpha$ cut is very important. The following results of algebraic operations for two triangular fuzzy numbers are well-known.

Example $2.7([2])$. Let $A=(1,2,4)$ and $B=(2,4,5)$ be triangular fuzzy numbers, i.e.,

$$
\mu_{A}(x)=\left\{\begin{array}{cl}
0, & x<1,4 \leq x \\
x-1, & 1 \leq x<2 \\
-\frac{1}{2} x+2, & 2 \leq x<4
\end{array}\right.
$$

and

$$
\mu_{B}(x)=\left\{\begin{array}{cl}
0, & x<2, \quad 5 \leq x \\
\frac{1}{2} x-1, & 2 \leq x<4 \\
-x+5, & 4 \leq x<5
\end{array}\right.
$$

we calculate exactly the above four operations using $\alpha$-cuts.

Let $A_{\alpha}$ and $B_{\alpha}$ be the $\alpha$-cuts of $A$ and $B$, respectively. Let $A_{\alpha}=\left[a_{1}^{(\alpha)}, a_{2}^{(\alpha)}\right]$ and $B_{\alpha}=\left[b_{1}^{(\alpha)}, b_{2}^{(\alpha)}\right]$. Since $\alpha=a_{1}^{(\alpha)}-1$ and $\alpha=-\frac{a_{2}^{(\alpha)}}{2}+2$, we have $A_{\alpha}=\left[a_{1}^{(\alpha)}, a_{2}^{(\alpha)}\right]=[\alpha+1,-2 \alpha+4]$. Since $\alpha=\frac{b_{1}^{(\alpha)}}{2}-1$ and $\alpha=-b_{2}^{(\alpha)}+5$, $B_{\alpha}=\left[b_{1}^{(\alpha)}, b_{2}^{(\alpha)}\right]=[2 \alpha+2,-\alpha+5]$.

(1) extended addition:

By the above facts, $A_{\alpha}(+) B_{\alpha}=\left[a_{1}^{(\alpha)}+b_{1}^{(\alpha)}, a_{2}^{(\alpha)}+b_{2}^{(\alpha)}\right]=[3 \alpha+3,-3 \alpha+9]$. Thus $\mu_{A(+) B}(x)=0$ on the interval $[3,9]^{c}$ and $\mu_{A(+) B}(x)=1$ at $x=6$. By the routine calculation, we have

$$
\mu_{A(+) B}(x)=\left\{\begin{array}{cl}
0, & x<3, \quad 9 \leq x, \\
\frac{1}{3} x-1, & 3 \leq x<6, \\
-\frac{1}{3} x+3, & 6 \leq x<9,
\end{array}\right.
$$

i.e., $A(+) B=(3,6,9)$.

(2) extended subtraction:

Since $A_{\alpha}(-) B_{\alpha}=\left[a_{1}^{(\alpha)}-b_{2}^{(\alpha)}, a_{2}^{(\alpha)}-b_{1}^{(\alpha)}\right]=[2 \alpha-4,-4 \alpha+2]$, we have $\mu_{A(-) B}(x)=0$ on the interval $[-4,2]^{c}$ and $\mu_{A(-) B}(x)=1$ at $x=-2$. By the 
routine calculation, we have

$$
\mu_{A(-) B}(x)=\left\{\begin{array}{cl}
0, & x<-4,2 \leq x, \\
\frac{1}{2} x+2, & -4 \leq x<-2, \\
-\frac{1}{4} x+\frac{1}{2}, & -2 \leq x<2,
\end{array}\right.
$$

i.e., $A(-) B=(-4,-2,2)$.

(3) extended multiplication:

Since $A_{\alpha}(\cdot) B_{\alpha}=\left[a_{1}^{(\alpha)} \cdot b_{1}^{(\alpha)}, a_{2}^{(\alpha)} \cdot b_{2}^{(\alpha)}\right]=\left[2 \alpha^{2}+4 \alpha+2,2 \alpha^{2}-14 \alpha+20\right]$, $\mu_{A(\cdot) B}(x)=0$ on the interval $[2,20]^{c}$ and $\mu_{A(\cdot) B}(x)=1$ at $x=8$. By the routine calculation, we have

$$
\mu_{A(\cdot) B}(x)=\left\{\begin{array}{cl}
0, & x<2, \quad 20 \leq x \\
\frac{-2+\sqrt{2 x}}{2}, & 2 \leq x<8 \\
\frac{7-\sqrt{9+2 x}}{2}, & 8 \leq x<20 .
\end{array}\right.
$$

Thus $A(\cdot) B$ is not a triangular fuzzy number.

(4) extended division:

Since $A_{\alpha}(/) B_{\alpha}=\left[\frac{a_{1}^{(\alpha)}}{b_{2}^{(\alpha)}}, \frac{a_{2}^{(\alpha)}}{b_{1}^{(\alpha)}}\right]=\left[\frac{\alpha+1}{-\alpha+5}, \frac{-\alpha+2}{\alpha+1}\right], \mu_{A(/) B}(x)=0$ on the interval $\left[\frac{1}{5}, 2\right]^{c}$ and $\mu_{A(/) B}(x)=1$ at $x=\frac{1}{2}$. By the routine calculation, we have

$$
\mu_{A(/) B}(x)=\left\{\begin{array}{cl}
0, & x<\frac{1}{5}, \quad 2 \leq x, \\
\frac{5 x-1}{x+1}, & \frac{1}{5} \leq x<\frac{1}{2}, \\
\frac{-x+2}{x+1}, & \frac{1}{2} \leq x<2 .
\end{array}\right.
$$

Thus $A(/) B$ is not a triangular fuzzy number.

Generally, for two triangular fuzzy numbers $A$ and $B, A(+) B$ and $A(-) B$ always become triangular fuzzy numbers. But $A(\cdot) B$ and $A(/) B$ may not be triangular fuzzy numbers.

\section{Main results}

In this section, we study a fuzzy number defined on $\mathbb{R}$. If $A$ is a fuzzy number on $\mathbb{R}$, the membership function $\mu_{A}(x)$ is piecewise continuous. We will find some piecewise continuous function $f_{\alpha}(t)$ such that the $\alpha$-cut $A_{\alpha}$ of $A$ equals to $\left\{f_{\alpha}(t) \mid t \in[0,1]\right\}$ in Theorem 3.2. Using $f_{\alpha}(t)$, we define parametric operations. And then we have the same results in Theorem 3.5 as the extended operations.

Definition 3.1. A $\alpha$-cut of a fuzzy number $A$ is defined by $A_{\alpha}=\{x \in \mathbb{R} \mid$ $\left.\mu_{A}(x) \geq \alpha\right\}$ if $\alpha \in(0,1]$ and $A_{\alpha}=\mathrm{cl}\left\{x \in \mathbb{R} \mid \mu_{A}(x)>\alpha\right\}$ if $\alpha=0$.

Note that a piecewise continuous function $f$ on $[a, b] \in \mathbb{R}$ means that the function $f$ is continuous on $[a, b]$ except finitely many points (it may contains $a$ or $b)$ in $[a, b]$. 
Theorem 3.2. Let $A$ be a fuzzy number defined on $\mathbb{R}$ and $A_{\alpha}=\{x \in A \mid$ $\left.\mu_{A}(x) \geq \alpha\right\}$ be a $\alpha$-cut of $A$. Then for all $\alpha \in[0,1]$, there exists a piecewise continuous function $f_{\alpha}(t)$ defined on $[0,1]$ such that $A_{\alpha}=\left\{f_{\alpha}(t) \mid t \in[0,1]\right\}$.

Proof. Since $A$ is a fuzzy number defined on $\mathbb{R}$, the membership function $\mu_{A}(x)$ is piecewise continuous. Let $A_{0}=[a, b]$ be the 0 -cut of $A$. Then $\mu_{A}(x)$ is continuous on $[a, b]$ except finitely many points $x_{1}<x_{2}<\cdots<x_{n}$. Note that $x_{1}$ and $x_{n}$ may equal to the end points $a$ and $b$, respectively. Let $\alpha \in[0,1]$ be fixed. Let $a_{1}^{(\alpha)}$ and $a_{2}^{(\alpha)}$ be the left and right end points of $A_{\alpha}$, respectively. Assume that $x_{1}<\cdots<x_{i}<a_{1}^{(\alpha)}<x_{i+1}<\cdots<x_{i+m}<a_{2}^{(\alpha)}<x_{i+m+1}<$ $\cdots<x_{n}$. If the end points $a_{1}^{(\alpha)}$ and $a_{2}^{(\alpha)}$ (or one of them) equal to some $x_{i}$, it can be proved similarly. Define

$$
f_{\alpha}(t)=\left(a_{2}^{(\alpha)}-a_{1}^{(\alpha)}\right) t+a_{1}^{(\alpha)} \quad \text { if } t \in[0,1],
$$

except the points

$$
t=\frac{x_{i+j}-a_{1}^{(\alpha)}}{a_{2}^{(\alpha)}-a_{1}^{(\alpha)}}, j=1,2, \ldots, m .
$$

Then $f_{\alpha}(t)$ is piecewise continuous on $[0,1]$ and $A_{\alpha}=\left\{f_{\alpha}(t) \mid t \in[0,1]\right\}$. In fact, if $x \in A_{\alpha}, \mu_{A}(x) \geq \alpha$ and $x \neq x_{i}(i=1,2, \ldots, n)$. Thus $a_{1}^{(\alpha)} \leq x \leq a_{2}^{(\alpha)}$. If $x=a_{1}^{(\alpha)}$ or $x=a_{2}^{(\alpha)}, f_{\alpha}(0)=a_{1}^{(\alpha)}$ or $f_{\alpha}(1)=a_{2}^{(\alpha)}$. If $a_{1}^{(\alpha)}<x<a_{2}^{(\alpha)}$, we have

$$
0<\frac{x-a_{1}^{(\alpha)}}{a_{2}^{(\alpha)}-a_{1}^{(\alpha)}}<1
$$

Let

$$
t=\frac{x-a_{1}^{(\alpha)}}{a_{2}^{(\alpha)}-a_{1}^{(\alpha)}} .
$$

Then $t \in(0,1)$ and $f_{\alpha}(t)=x$. Thus $x \in\left\{f_{\alpha}(t) \mid t \in[0,1]\right\}$. This proves that $A_{\alpha} \subset\left\{f_{\alpha}(t) \mid t \in[0,1]\right\}$. Let $x=f_{\alpha}(t)=\left(a_{2}^{(\alpha)}-a_{1}^{(\alpha)}\right) t+a_{1}^{(\alpha)}$ for some $t \in[0,1]$ except $t=\frac{x_{i+j}-a_{1}^{(\alpha)}}{a_{2}^{(\alpha)}-a_{1}^{(\alpha)}}, \quad j=1,2, \ldots, m$. Then $a_{1}^{(\alpha)} \leq x \leq a_{2}^{(\alpha)}$ and $x \neq x_{i}(i=1,2, \ldots, n)$. Thus $\mu_{A}(x) \geq \alpha$ and $x \in A_{\alpha}$. The proof is complete.

We call a fuzzy number $A$ is continuous if the membership function $\mu_{A}(x)$ of $A$ is continuous. If $A$ is a continuous fuzzy number, then the $\alpha$-cut $A_{\alpha}$ of $A$ is a closed interval in $\mathbb{R}$.

Corollary 3.3. Let $A$ be a continuous fuzzy number defined on $\mathbb{R}$. Then the $\alpha$-cut $A_{\alpha}=\left\{x \in A \mid \mu_{A}(x) \geq \alpha\right\}$ becomes a closed interval $\left[a_{1}^{(\alpha)}, a_{2}^{(\alpha)}\right]$ on $\mathbb{R}$. And for all $\alpha \in[0,1]$, there exists a continuous function $f_{\alpha}(t)$ defined on $[0,1]$ such that $\left[a_{1}^{(\alpha)}, a_{2}^{(\alpha)}\right]=\left\{f_{\alpha}(t) \mid t \in[0,1]\right\}$. 
The above corresponding function $f_{\alpha}(t)$ is said to be the parametric $\alpha$ function of $A$. And the parametric $\alpha$-function of $A$ is denoted by $f_{\alpha}(t)$ or $f_{A}(t)$.

Definition 3.4. Let $A$ and $B$ be two continuous fuzzy numbers defined on $\mathbb{R}$ and $A_{\alpha}, B_{\alpha}, f_{A}(t), f_{B}(t)$ be the $\alpha$-cuts and parametric $\alpha$-functions of $\mathrm{A}$ and $\mathrm{B}$, respectively. The parametric addition, parametric subtraction, parametric multiplication and parametric division are fuzzy numbers which have their $\alpha$ cuts as followings.

(1) parametric addition $A(+)_{p} B$ :

$$
\left(A(+)_{p} B\right)_{\alpha}=\left\{f_{A}(t)+f_{B}(t) \mid t \in[0,1]\right\} .
$$

(2) parametric subtraction $A(-)_{p} B$ :

$$
\left(A(-)_{p} B\right)_{\alpha}=\left\{f_{A}(t)-f_{B}(1-t) \mid t \in[0,1]\right\} .
$$

(3) parametric multiplication $A(\cdot)_{p} B$ :

$$
\left(A(\cdot)_{p} B\right)_{\alpha}=\left\{f_{A}(t) \cdot f_{B}(t) \mid t \in[0,1]\right\} .
$$

(4) parametric division $A(/)_{p} B$ :

$$
\left(A(/)_{p} B\right)_{\alpha}=\left\{f_{A}(t) / f_{B}(1-t) \mid t \in[0,1]\right\} .
$$

Theorem 3.5. Let $A$ and $B$ be two continuous fuzzy numbers defined on $\mathbb{R}$. Then we have the followings.
(1) $A(+)_{p} B=A(+) B$.
(2) $A(-)_{p} B=A(-) B$.
(3) $A(\cdot)_{p} B=A(\cdot) B$.
(4) $A(/)_{p} B=A(/) B$.

Proof. Let $A_{\alpha}=\left[a_{1}^{(\alpha)}, a_{2}^{(\alpha)}\right], B_{\alpha}=\left[b_{1}^{(\alpha)}, b_{2}^{(\alpha)}\right], f_{A}(t), f_{B}(t)$ be the $\alpha$-cuts and parametric $\alpha$-functions of $A$ and $B$, respectively. Then $f_{A}(0)=a_{1}^{(\alpha)}, f_{A}(1)=$ $a_{2}^{(\alpha)}, f_{B}(0)=b_{1}^{(\alpha)}$ and $f_{B}(1)=b_{2}^{(\alpha)}$. have

(1) Since $f_{A}(0)+f_{B}(0)=a_{1}^{(\alpha)}+b_{1}^{(\alpha)}$ and $f_{A}(1)+f_{B}(1)=a_{2}^{(\alpha)}+b_{2}^{(\alpha)}$, we

$$
\begin{aligned}
\left(A(+)_{p} B\right)_{\alpha} & =\left\{f_{A}(t)+f_{B}(t) \mid t \in[0,1]\right\} \\
& =\left[a_{1}^{(\alpha)}+b_{1}^{(\alpha)}, a_{2}^{(\alpha)}+b_{2}^{(\alpha)}\right] \\
& =(A(+) B)_{\alpha} .
\end{aligned}
$$

Thus $A(+)_{p} B=A(+) B$.

(2) Since $f_{A}(0)-f_{B}(1)=a_{1}^{(\alpha)}-b_{2}^{(\alpha)}$ and $f_{A}(1)-f_{B}(0)=a_{2}^{(\alpha)}-b_{1}^{(\alpha)}$, we have

$$
\begin{aligned}
\left(A(-)_{p} B\right)_{\alpha} & =\left\{f_{A}(t)-f_{B}(1-t) \mid t \in[0,1]\right\} \\
& =\left[a_{1}^{(\alpha)}-b_{2}^{(\alpha)}, a_{2}^{(\alpha)}-b_{1}^{(\alpha)}\right] \\
& =(A(-) B)_{\alpha} .
\end{aligned}
$$


Thus $A(-)_{p} B=A(-) B$.

(3) Since $f_{A}(0) \cdot f_{B}(0)=a_{1}^{(\alpha)} \cdot b_{1}^{(\alpha)}$ and $f_{A}(1) \cdot f_{B}(1)=a_{2}^{(\alpha)} \cdot b_{2}^{(\alpha)}$, we have

$$
\begin{aligned}
\left(A(\cdot)_{p} B\right)_{\alpha} & =\left\{f_{A}(t) \cdot f_{B}(t) \mid t \in[0,1]\right\} \\
& =\left[a_{1}^{(\alpha)} \cdot b_{1}^{(\alpha)}, a_{2}^{(\alpha)} \cdot b_{2}^{(\alpha)}\right] \\
& =(A(\cdot) B)_{\alpha} .
\end{aligned}
$$

Thus $A(\cdot)_{p} B=A(\cdot) B$.

(4) Since $f_{A}(0) / f_{B}(1)=a_{1}^{(\alpha)} / b_{2}^{(\alpha)}$ and $f_{A}(1) / f_{B}(0)=a_{2}^{(\alpha)} / b_{1}^{(\alpha)}$, we have

$$
\begin{aligned}
\left(A(/)_{p} B\right)_{\alpha} & =\left\{f_{A}(t) / f_{B}(1-t) \mid t \in[0,1]\right\} \\
& =\left[a_{1}^{(\alpha)} / b_{2}^{(\alpha)}, a_{2}^{(\alpha)} / b_{1}^{(\alpha)}\right] \\
& =(A(/) B)_{\alpha} .
\end{aligned}
$$

Thus $A(/)_{p} B=A(/) B$.

Corollary 3.6. Let $A$ and $B$ be two triangular fuzzy numbers defined on $\mathbb{R}$. Then we have the followings.

(1) $A(+)_{p} B=A(+) B$.

(2) $A(-)_{p} B=A(-) B$.

(3) $A(\cdot)_{p} B=A(\cdot) B$

(4) $A(/)_{p} B=A(/) B$.

Example 3.7. In Example 2.7, the $\alpha$-cuts of two triangular fuzzy numbers $A=$ $(1,2,4)$ and $B=(2,4,5)$ are $A_{\alpha}=[\alpha+1,-2 \alpha+4]$ and $B_{\alpha}=[2 \alpha+2,-\alpha+5]$, respectively. In this case, $A_{\alpha}=f_{A}(t)=\{(-3 \alpha+3) t+\alpha+1 \mid t \in[0,1]\}$ and $B_{\alpha}=f_{B}(t)=\{(-3 \alpha+3) t+2 \alpha+2 \mid t \in[0,1]\}$. Thus we have the followings.

(1) $\left(A(+)_{p} B\right)_{\alpha}=\{(-6 \alpha+6) t+3 \alpha+3 \mid t \in[0,1]\}$.

(2) $\left(A(-)_{p} B\right)_{\alpha}=\{(-6 \alpha+6) t+2 \alpha-4 \mid t \in[0,1]\}$.

(3) $\left(A(\cdot)_{p} B\right)_{\alpha}=\{((-3 \alpha+3) t+\alpha+1) \cdot((-3 \alpha+3) t+2 \alpha+2) \mid t \in[0,1]\}$.

(4) $\left(A(/)_{p} B\right)_{\alpha}=\left\{\frac{(-3 \alpha+3) t+\alpha+1}{(3 \alpha-3) t-\alpha+5} \mid t \in[0,1]\right\}$.

We have the same $\alpha$-cuts as those in Example 2.7. In fact,

$$
\begin{aligned}
A_{\alpha}(+) B_{\alpha} & =\left[a_{1}^{(\alpha)}+b_{1}^{(\alpha)}, a_{2}^{(\alpha)}+b_{2}^{(\alpha)}\right] \\
& =[3 \alpha+3,-3 \alpha+9], \\
A_{\alpha}(-) B_{\alpha} & =\left[a_{1}^{(\alpha)}-b_{2}^{(\alpha)}, a_{2}^{(\alpha)}-b_{1}^{(\alpha)}\right] \\
& =[2 \alpha-4,-4 \alpha+2], \\
A_{\alpha}(\cdot) B_{\alpha} & =\left[a_{1}^{(\alpha)} \cdot b_{1}^{(\alpha)}, a_{2}^{(\alpha)} \cdot b_{2}^{(\alpha)}\right] \\
& =\left[2 \alpha^{2}+4 \alpha+2,2 \alpha^{2}-14 \alpha+20\right]
\end{aligned}
$$


and

$$
A_{\alpha}(/) B_{\alpha}=\left[\frac{a_{1}^{(\alpha)}}{b_{2}^{(\alpha)}}, \frac{a_{2}^{(\alpha)}}{b_{1}^{(\alpha)}}\right]=\left[\frac{\alpha+1}{-\alpha+5}, \frac{-\alpha+2}{\alpha+1}\right] .
$$

\section{References}

[1] A. Kaufmann and M. M. Gupta, Introduction To Fuzzy Arithmetic, Theory and Applications, Van Nostrand Reinhold Co., New York, 1985.

2] Y. S. Yun, S. U. Ryu, and J. W. Park, The generalized triangular fuzzy sets, J. Chungcheong Math. Soc. 22 (2009), no. 2, 161-170.

[3] L. A. Zadeh, The concept of a linguistic variable and its application to approximate reasoning. I, Inform. Sci. 8 (1975), 199-249.

[4] H. J. Zimmermann, Fuzzy Set Theory - and Its Applications, Kluwer-Nijhoff Publishing, 1985.

JisOO BYUN

Department of Mathematics Education

KyUngnam University

Gyeongsangnam-Do 631-701, Korea

E-mail address: jisoobyun@kyungnam.ac.kr

YONG SIK YUN

Department of Mathematics

AND

Research Institute for Basic Sciences

Jeju National University

Jeju 690-756, Korea

E-mail address: yunys@jejunu.ac.kr 